\title{
Efficient Outage Probability Evaluation of Diversity Receivers Over $\alpha-\mu$ Fading Channels
}

\author{
Chaouki Ben Issaid, Mohamed-Slim Alouini, and Raul Tempone \\ King Abdullah University of Science and Technology (KAUST), \\ Computer, Electrical, and Mathematical Sciences and Engineering (CEMSE) Division, \\ Thuwal, Makkah Province, Saudi Arabia \\ Email: \{chaouki.benissaid, slim.alouini, raul.tempone\}@kaust.edu.sa
}

\begin{abstract}
In this paper, we are interested in determining the cumulative distribution function of the sum of $\alpha-\mu$ random variables in the setting of rare event simulations. To this end, we present an efficient importance sampling estimator. The main result of this work is the bounded relative error property of the proposed estimator. This result is used to accurately estimate the outage probability of multibranch maximum ratio combining and equal gain diversity receivers over $\alpha-\mu$ fading channels. Selected numerical simulations are discussed to show the robustness of our estimator compared to naive Monte Carlo.
\end{abstract}

Index Terms $-\alpha-\mu$, importance sampling, Monte Carlo, bounded relative error, outage probability, diversity techniques.

\section{INTRODUCTION}

The $\alpha-\mu$ distribution [1] is a flexible distribution that includes many popular fading models such as Weibull or Nakagami-m as special cases. It has the same functional form as the well-known generalized Gamma (GG) distribution [2]. According to [1], the $\alpha-\mu$ distribution can be used to model the small-scale variation of the fading signal in non-line-of sight wireless radio-frequency channels. The reader is referred to [1] for the physical interpretation of the $\alpha-\mu$ fading model and to [2] for the mathematical formulation of the GG distribution.

Diversity techniques are often used to reduce the fading caused by multipath transmission channel [3, Chap. 9]. They rely on receiving multiple transmitted signal replicas affected by independent fadings. When some of these techniques are considered, one of the main challenges in evaluating the system performance is that the sum of the fading envelopes or powers is involved. Although the study of diversity receivers for many important fading channels received a great deal of attention, only few works investigated the performance of diversity receivers over $\alpha-\mu$ fading channels. This amounts to finding the distribution of the sum of $\alpha-\mu$ variates. Stacy managed in his seminal work [2] to derive an infinite series representation of the cumulative distribution function (CDF) of the sum of GG variates. However, the author points out that such representation presents certain computational challenges. Most of the other attempts fail to provide a closed-form expression for this cumbersome problem and only propose to tackle it by deriving approximate solutions that usually involve a truncation error. In [4], Piboongungon et al. investigated the average symbol error rate (SER) performance of both maximum ratio combining (MRC) and equal gain combining (EGC) receivers over GG fading channels. The authors used the moment generation function (MGF)-based approach [3] in the case of MRC receivers and the characteristic function (CHF)-based approach [5] for the EGC receivers. In [6], Sagias et al. proposed an upper bound for the sum of GG random variables (RVs) for the purpose of studying the performance of EGC receivers. First, they derived the expression of the CDF of the product of GG variates. Then, by exploiting the well-known inequality between the arithmetic and geometric means, the authors were able to provide an upper bound for the problem of the sum. Later on, Da Costa et al. presented a highly accurate closed-form approximations to the probability density function (PDF) and CDF of the sum of independent identically distributed (i.i.d.) $\alpha-\mu$ variates [7]. In this work, the authors approximate the sum of i.i.d $\alpha-\mu \mathrm{RV}$ s by one $\alpha-\mu$ $\mathrm{RV}$. To determine the parameters $\alpha$ and $\mu$ of the approximate distribution, a moment-based method was introduced. This result was used to approximate the outage probability as well as the average bit error probability for EGC and MRC diversity techniques. Although numerical simulations show a good agreement between the approximate and the exact solution, the proposed approach is restricted to the i.i.d case. Recently, closed-form expressions for the SER of EGC and MRC receivers over $\alpha-\mu$ fading were derived in [8]. Using the Mellin transform, El Ayadi et al. expressed the SER in terms of the Fox H-functions.

In summary, determining an exact expression for the outage probability is a challenging task in many cases, and to the best of our knowledge, no closed-form results for the outage probability of multibranch diversity receivers operating over $\alpha-\mu$ fading channels were derived in the literature. In this case, the only way to study the system performance is by means of numerical simulations, e.g. using Monte Carlo (MC) method. Due to the simplicity of its implementation, MC can be seen as a powerful technique when the problem is too complex and difficult to be solved analytically. However, it proves its inefficiency to estimate a rare event probability, i.e. probability lower than $10^{-8}$, since too many samples are needed to guarantee a good quality estimator. To solve this problem, many accelerated simulation methods have been proposed in the literature. Recently, some efforts have been made to propose efficient estimators to evaluate 
the outage probability with diversity techniques for wireless communication systems. For instance, the authors in [9] have addressed this problem using two unified importance sampling (IS) schemes. However, the work was limited to the i.i.d case. Later on, the authors proposed in [10] a mean-shift IS scheme that evaluates the outage probability of multibranch MRC diversity receivers over Gamma-Gamma fading channels in both the i.i.d and independent not necessarily identically distributed (i.n.i.d). While the approach proposed in [10] does not necessarily work with all types of fading distributions, it can work with the types of fading distributions for which one can identify a parameter in the functional form of the PDF that has an effect on the mean (by shifting it towards the origin) or on the variance (by scaling it). For instance, we show in this paper, how this approach can be used to estimate accurately the left tail probability of sum of i.n.i.d $\alpha-\mu$ variates, a problem for which no exact solution has been proposed so far to our best knowledge. Interestingly enough, we were also able to prove that the bounded relative error property of the IS estimator still holds in this kind of problem, resulting in a significant reduction in terms of number of simulation runs.

The rest of this paper is organized as follows. First, we describe the problem setting in Section II. Then, we give a brief description of the IS method in Section III. In section IV, we present our approach to estimate the outage probability for diversity receivers over $\alpha-\mu$ fading channels as well as the main theorem proving the efficiency of the proposed method in the i.n.i.d case. Prior to concluding, we show, in Section V, some selected simulation results related to the evaluation of the outage probability of multibranch diversity receivers over $\alpha-\mu$ fading channels. We also compare the computational efficiency of our approach compared to naive MC.

\section{SySTEM MOdeL}

The instantaneous signal-to-noise ratio (SNR) expression at the diversity receiver, is given by [11]

$$
\gamma_{\text {end }}=\frac{E_{s}}{N_{0} \sqrt{L^{1-p+q}}}\left(\sum_{\ell=1}^{L} X_{\ell}^{p}\right)^{q},
$$

where $(p, q)=(1,2)$ for the EGC case and $(p, q)=(2,1)$ for the MRC case. The ratio $\frac{E_{s}}{N_{0}}$ is the SNR per symbol at the transmitter, $L$ is the number of diversity branches, and $\left\{X_{\ell}\right\}_{\ell=1}^{L}$ are the channel gains which are modeled as i.n.i.d $\alpha-\mu$ RVs with parameters $\left(\alpha_{\ell}, \mu_{\ell}, \Omega_{\ell}\right), \ell=1, \ldots, L$, whose PDFs are given by [1, Eq. (1)]

$$
f_{X_{\ell}}(x)=\frac{\alpha_{\ell} \mu_{\ell}^{\mu_{\ell}} x^{\alpha_{\ell} \mu_{\ell}-1}}{\Omega_{\ell}^{\mu_{\ell}} \Gamma\left(\mu_{\ell}\right)} \exp \left(-\frac{\mu_{\ell}}{\Omega_{\ell}} x^{\alpha_{\ell}}\right), x \geq 0,
$$

where $\alpha_{\ell}$ and $\mu_{\ell}$ are two positive real numbers that represent the distribution shape parameters, $\Gamma(\cdot)$ is the Gamma function [12, Sec. (8.31)], and $\Omega_{\ell}$ is linked to the mean of $X_{\ell}$ as

$$
\mathbb{E}\left[X_{\ell}\right]=\left(\frac{\Omega_{\ell}}{\mu_{\ell}}\right)^{\frac{1}{\alpha_{\ell}}} \frac{\Gamma\left(\mu_{\ell}+\frac{1}{\alpha_{\ell}}\right)}{\Gamma\left(\mu_{\ell}\right)}, \ell=1, \ldots, L .
$$

The $\alpha-\mu$ (or $\mathrm{GG}$ ) distribution is a generic model that covers Weibull, Gamma, Nakagami-m and other distributions as special cases. Table I summarizes these special cases when one or more of the GG distribution parameters is set equal to specific values.

TABLE I

SPECIAL CASES OF THE $\alpha-\mu$ DISTRIBUTION.

\begin{tabular}{l|c|c|c}
\hline Distribution & $\alpha$ & $\mu$ & $\Omega$ \\
\hline \hline Chi-squared & 1 & $\in \mathbb{N} \backslash\{0\}$ & $2 m$ \\
\hline Erlang & 1 & $\in \mathbb{N} \backslash\{0\}$ & - \\
\hline Exponential & 1 & 1 & - \\
\hline Rayleigh & 2 & 1 & - \\
\hline One-sided Gaussian & 2 & $\frac{1}{2}$ & - \\
\hline Gamma & 1 & - & - \\
\hline Nakagami-m & 2 & - & - \\
\hline Weibull & - & 1 & - \\
\hline
\end{tabular}

The quality of a communication system can be evaluated by computing the outage probability. This metric is a function of the transmission technique used, but also the channel on which the signal is transmitted. More specifically, for a given threshold $\gamma_{t h}$, the outage probability $P$ is defined as the probability that the instantaneous SNR drops below $\gamma_{t h}$, i.e.

$$
P=\mathbb{P}\left(\gamma_{\text {end }} \leq \gamma_{\text {th }}\right)=\mathbb{P}\left(\sum_{\ell=1}^{L} X_{\ell}^{p} \leq\left(\frac{N_{0}}{E_{s}} \sqrt{L^{1-p+q}} \gamma_{t h}\right)^{\frac{1}{q}}\right) .
$$

In the remainder of the paper, we will focus on the EGC case and we will show how the approach can be easily extended to the MRC case. Thereby, unless stated otherwise, $P$ will be given by

$$
P=\mathbb{P}\left(S_{L}=\sum_{\ell=1}^{L} X_{\ell} \leq \gamma_{0}=\left(\frac{N_{0} L}{E_{s}} \gamma_{t h}\right)^{\frac{1}{2}}\right) .
$$

We can see that our goal of estimating the outage probability reduces to finding the CDF of the sum of $\alpha-\mu$ RVs. In particular, we are interested in the case where the outage probability requirements are very low, i.e. in the range of $10^{-6}$ to $10^{-10}$. For instance, this is common in areas such as wireless back-hauling using free space optics (FSO) [13] and millimeter wave [14].

\section{IMPORTANCE SAMPLING}

The outage probability is given by $P=\mathbb{E}\left[\mathbb{1}_{\left(S_{L} \leq \gamma_{0}\right)}\right]$, where $\mathbb{E}[\cdot]$ is the expectation with respect to (w.r.t) the probability measure under which the PDF of $X_{\ell}$ is $f_{X_{\ell}}(\cdot)$, $\ell=1,2, \ldots, L$. The naive MC estimator of (4) is thus

$$
\hat{P}_{M C}=\frac{1}{N} \sum_{i=1}^{N} \mathbb{1}_{\left(S_{L}\left(\omega_{i}\right) \leq \gamma_{0}\right)},
$$

where $N$ is the number of MC samples, $\mathbb{1}_{(\cdot)}$ is the indicator function, and $\left\{S_{L}\left(\omega_{i}\right)\right\}_{i=1}^{N}$ are i.i.d. realizations of the RV $S_{L}$. The sequence $\left\{X_{\ell}\left(\omega_{i}\right)\right\}_{\ell=1}^{L}$ is sampled independently according to the PDFs (2) for each realization of $S_{L}$. 
By introducing new biased densities $\left\{f_{X_{\ell}}^{*}(\cdot)\right\}_{\ell=1}^{L}$, we can re-write $P=\mathbb{E}^{*}\left[\mathbb{1}_{\left(S_{L} \leq \gamma_{0}\right)} \mathcal{L}\left(X_{1}, \ldots, X_{L}\right)\right]$ where $\mathbb{E}^{*}[\cdot]$ denotes the expectation w.r.t the probability measure under which the PDF of $X_{\ell}$ is $f_{X_{\ell}}^{*}(\cdot), \ell=1,2, \ldots, L$.

The likelihood ratio $\mathcal{L}\left(X_{1}, \ldots, X_{L}\right)$ is defined as

$$
\mathcal{L}\left(X_{1}, \ldots, X_{L}\right)=\prod_{\ell=1}^{L} \frac{f_{X_{\ell}}\left(X_{\ell}\right)}{f_{X_{\ell}}^{*}\left(X_{\ell}\right)}
$$

In this case, the IS estimator of (4) is

$$
\hat{P}_{I S}=\frac{1}{N^{*}} \sum_{i=1}^{N^{*}} \mathbb{1}_{\left(S_{L}\left(\omega_{i}\right) \leq \gamma_{0}\right)} \mathcal{L}\left(X_{1}\left(\omega_{i}\right), \ldots, X_{L}\left(\omega_{i}\right)\right),
$$

where for each realization $i=1, \ldots, N$, the sequence $\left\{X_{\ell}\left(\omega_{i}\right)\right\}_{\ell=1}^{L}$ are sampled independently according to the biased PDFs $\left\{f_{\ell}^{*}(\cdot)\right\}_{\ell=1}^{L}$.

To assess the goodness of an IS approach, many criteria has been introduced in previous works (see, for instance, [15] and references therein) among them we find the bounded relative error, one of the desirable properties in the field of rare events algorithms.

\section{PROpOSED APPROACH}

In this section, a clever choice of the biased PDF is introduced. In fact, we propose to introduce the parameter $\Omega_{\ell}^{*}=\Omega_{\ell}-\Omega_{0, \ell}$ in the new biased PDF where $\Omega_{0, \ell}$ satisfies $0 \leq \Omega_{0, \ell}<\Omega_{\ell}$ and as $\gamma_{0} \rightarrow 0$, it approaches $\Omega_{\ell}, \ell=1, \ldots, L$. This choice is justified by the fact that the biased PDF belongs to the same family as the original PDF so the sampling from it should be simple. In this case, the biased PDF is

$$
\begin{aligned}
f_{X_{\ell}}^{*}(x) & =\frac{\alpha_{\ell} \mu_{\ell}^{\mu_{\ell}} x^{\alpha_{\ell} \mu_{\ell}-1}}{\left(\Omega_{\ell}-\Omega_{0, \ell}\right)^{\mu_{\ell}} \Gamma\left(\mu_{\ell}\right)} \exp \left(-\frac{\mu_{\ell} x^{\alpha_{\ell}}}{\Omega_{\ell}-\Omega_{0, \ell}}\right), x \geq 0 \\
\ell & =1, \ldots, L .
\end{aligned}
$$

For the selection of the parameters $\left\{\Omega_{0, \ell}\right\}_{\ell=1}^{L}$, we require that the equation $\mathbb{E}^{*}\left[S_{L}\right]=\gamma_{0}$ holds in order to encourage the sampling from the region of interest $\left\{S_{L} \leq \gamma_{0}\right\}$. Since this equation has infinitely many solutions, we choose a particular solution of the following form

$$
\Omega_{0, \ell}=\Omega_{\ell}-\beta_{\ell} \gamma_{0}^{\alpha_{\ell}}, \forall \ell=1, \ldots L,
$$

where $\beta_{\ell}=\left[\frac{\mu_{\ell} \Gamma\left(\mu_{\ell}\right)}{\Gamma\left(\mu_{\ell}+\frac{1}{\alpha_{\ell}}\right) L}\right]^{\alpha_{\ell}}$.

The following theorem characterizes the efficiency of our proposed IS estimator.

Theorem 1. Let $\left\{X_{\ell}\right\}_{\ell=1}^{L}$ be a sequence of i.n.i.d $\alpha-\mu \mathrm{RVs}$ and $f_{X_{\ell}}^{*}(\cdot)$ be defined as in (8) where $\Omega_{0, \ell}$ is given by (9). Then, the IS estimator (7) has a bounded relative error, i.e.

$$
\limsup _{\gamma_{0} \rightarrow 0} \frac{\mathbb{E}^{*}\left[\mathbb{1}_{\left(S_{L} \leq \gamma_{0}\right)} \mathcal{L}^{2}\left(X_{1}, \ldots, X_{L}\right)\right]}{P^{2}}<+\infty .
$$

provided that $\min _{1 \leq \ell \leq L} \alpha_{\ell}>1$.

Proof: See Appendix A.
Remark 1. To accurately estimate the probability $P$, naive MC requires a number of samples of the order of $\mathcal{O}\left(P^{-1}\right)$. However, for the same accuracy requirement and when the IS estimator is endowed with the bounded relative error property, the number of simulation runs $N$ required remains bounded independently of how small the outage probability $P$ is.

Remark 2. In this remark, we show briefly how our approach is easily extendable to the MRC case. First, we recall the expression of the outage probability in this case

$$
P=\mathbb{P}\left(\sum_{\ell=1}^{L} X_{\ell}^{2} \leq \eta_{0}=\frac{N_{0}}{E_{s}} \gamma_{t h}\right)
$$

where $X_{\ell}^{2}$ is also a $\alpha-\mu \mathrm{RV}$ with parameters $\left(\frac{\alpha_{\ell}}{2}, \mu_{\ell}, \Omega_{\ell}\right)$. As we can see from (11), the problem is again reduced to finding the CDF of the sum of $\alpha-\mu$ variates. Using the approach described in this section, we can easily evaluate the outage probability given by (11) for the MRC scenario.

To compare the efficiency of IS to naive MC, we need to compare the number of simulation runs required by each method to achieve the same accuracy requirement $\varepsilon$. To this end, we introduce the relative error of naive MC simulation

$$
\varepsilon=\frac{C}{P} \sqrt{\frac{P(1-P)}{N}},
$$

where $C=1.96$ which corresponds to a $95 \%$ confidence interval. Similarly, we define the relative error of the IS method

$$
\varepsilon^{*}=\frac{C}{P} \sqrt{\frac{\mathbb{V}^{*}\left[\mathbb{1}_{\left(S_{L} \leq \gamma_{0}\right)} \mathcal{L}\left(X_{1}, \ldots, X_{L}\right)\right]}{N}} .
$$

Let $\epsilon_{0}$ be a fixed accuracy requirement. Using Eqs. (12) and (13), we can determine the number of samples needed by naive $\mathrm{MC}$ and IS simulations respectively

$$
\begin{aligned}
N & =P(1-P)\left(\frac{C}{P \epsilon_{0}}\right)^{2}, \\
N^{*} & =\mathbb{V}^{*}\left[\mathbb{1}_{\left(S_{L} \leq \gamma_{0}\right)} \mathcal{L}\left(X_{1}, \ldots, X_{L}\right)\right]\left(\frac{C}{P \epsilon_{0}}\right)^{2} .
\end{aligned}
$$

\section{Simulation Results}

This section presents the numerical simulations regarding the estimation of the outage probability using both naive MC and our proposed IS method. The accuracy, as well as the efficiency, of both methods is analyzed. Table II details the fading parameters $\left(\alpha_{\ell}, \mu_{\ell}\right)$ used in this section for two scenarios $L=4$ and $L=6$. In Fig. 1, we plot the outage probability $P$ against the SNR threshold $\gamma_{t h}$ using naive MC (blue curve) and our proposed IS approach (red curve). The solid line represents the EGC scenario while the dashed line is for the MRC case. Similar conclusions can be drawn for both type of diversity. In fact, we notice that for the range of probabilities between $10^{-1}$ and $10^{-4}$, both methods match for the two cases. However, as the probability becomes smaller, naive MC fails to estimate the outage probability with the same accuracy as our method. In fact, we can see that for $L=6$, naive $\mathrm{MC}$ with $N=10^{7}$ samples is unable to 
estimate accurately the outage probabilities below $10^{-6}$ while the proposed IS scheme can estimate $P$ even with a small number of samples $N^{*}=10^{4}$.

TABLE II

FADING PARAMETERS USED TO SIMULATE THE OUTAGE PROBABILITY OF $L$-BRANCH DIVERSITY RECEIVERS OVER I.N.I.D $\alpha-\mu$ FADING MODEL.

\begin{tabular}{c|c}
\hline$L$ & Fading Parameters $\left(\alpha_{\ell}, \mu_{\ell}\right)$ \\
\hline \hline 4 & $(1.5,2),(2,2.5),(2.2,3.2),(1.5,3.5)$ \\
\hline 6 & $(1.5,3),(1.8,2.5),(2.5,2.8),(2.2,3),(1.5,3.25),(1.2,2.5)$ \\
\hline
\end{tabular}

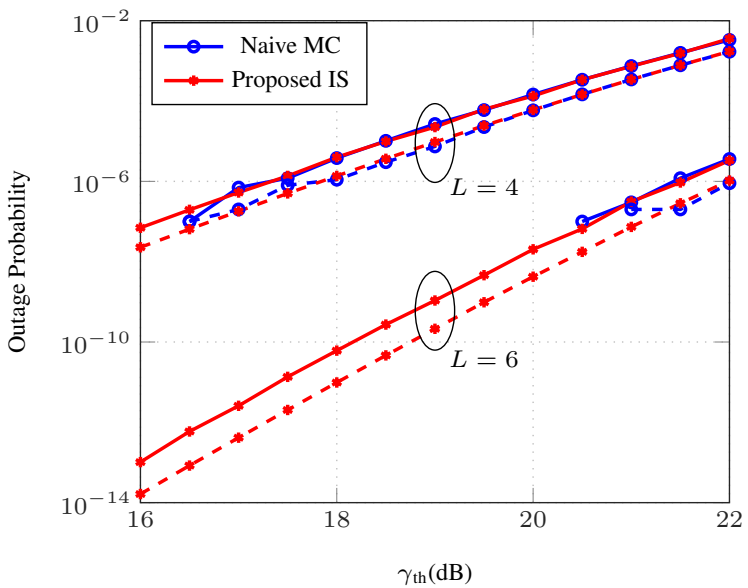

Fig. 1. Outage probability of $L$-branch diversity receivers over $\alpha-\mu$ fading model with $E_{s} / N_{0}=10 \mathrm{~dB}$ and $\Omega=10 \mathrm{~dB}$. Number of samples $N=10^{7}$ and $N^{*}=10^{4}$. EGC case: solid line and MRC case: dashed line.

The behavior of the number of required simulations runs by both methods for a fixed accuracy requirement $\varepsilon=\varepsilon^{*}=5 \%$ is depicted in Fig. 2 for the EGC case. We can observe that, for high outage probabilities, naive MC is sufficient for the estimation of $P$ since the number of simulation runs for both methods is quite the same. Our method outperforms naive MC in the region of rare events, i.e $P<10^{-6}$. In this region, the number of samples $N$ needed by $\mathrm{MC}$ to estimate $P$ up to $95 \%$ accuracy grows rapidly whereas $N^{*}$ remains almost constant. This goes hand in hand with the bounded relative error property of our IS estimator. To illustrate this idea, the number of samples $N^{*}$ required by IS, for $L=4$ is approximately $2.45 \times 10^{3}$ (respectively $1.2 \times 10^{5}$ ) times less than the number of samples used in MC simulations for $\gamma_{t h}$ $=18 \mathrm{~dB}$ (respectively $\gamma_{t h}=16 \mathrm{~dB}$ ). Similar conclusions can be drawn for the MRC scenario.

\section{CONCLUSION}

In this paper, a novel approach for the efficient estimation of the left tail of the sum of $\alpha-\mu$ variates is presented. We showed that our proposed estimator is endowed with the bounded relative error criterion. We were able to efficiently evaluate the outage probability of $L$-branch diversity receivers. Simulation results show the accuracy, as well as the efficiency, of our proposed IS estimator compared to the naive MC estimator. The proposed approach can be extended to other fading models, such as $\kappa-\mu$ and $\eta-\mu[16]$.

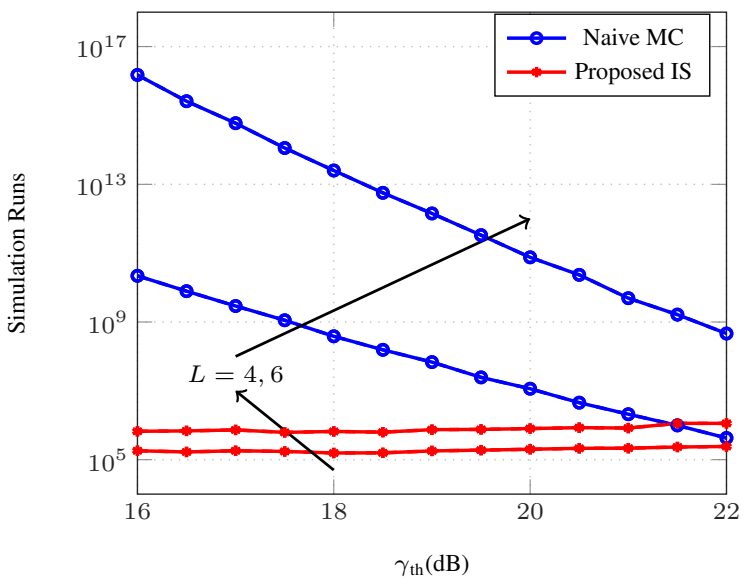

Fig. 2. Number of required simulation runs for $5 \%$ relative error for $L$ branch EGC diversity receivers over $\alpha-\mu$ fading model with $E_{s} / N_{0}=10$ $\mathrm{dB}$ and $\Omega=10 \mathrm{~dB}$.

\section{APPENDIX A \\ PROOF OF THEOREM 1}

Proof: The likelihood ratio is given by

$$
\begin{aligned}
& \mathcal{L}\left(X_{1}, \ldots, X_{L}\right)=\prod_{\ell=1}^{L} \frac{f_{X_{\ell}}\left(X_{\ell}\right)}{f_{X_{\ell}}^{*}\left(X_{\ell}\right)} \\
& =\prod_{\ell=1}^{L}\left(\frac{\Omega_{\ell}-\Omega_{0, \ell}}{\Omega_{\ell}}\right)^{\mu_{\ell}} \prod_{\ell=1}^{L} \exp \left(\mu_{\ell}\left[\frac{1}{\Omega_{\ell}-\Omega_{0, \ell}}-\frac{1}{\Omega_{\ell}}\right] x_{\ell}^{\alpha_{\ell}}\right) .
\end{aligned}
$$

Replacing the expression of $\Omega_{0, \ell}$ in (8), we get

$$
\begin{aligned}
& \mathcal{L}\left(X_{1}, \ldots, X_{L}\right)=\prod_{\ell=1}^{L}\left(\frac{\beta_{\ell} \gamma_{0}^{\alpha_{\ell}}}{\Omega_{\ell}}\right)^{\mu_{\ell}} \\
& \times \exp \left(\sum_{\ell=1}^{L} \mu_{\ell}\left[\frac{1}{\beta_{\ell} \gamma_{0}^{\alpha_{\ell}}}-\frac{1}{\Omega_{\ell}}\right] x_{\ell}^{\alpha_{\ell}}\right) .
\end{aligned}
$$

Thereby, the likelihood can be bounded by

$$
\begin{aligned}
& \mathcal{L}\left(X_{1}, \ldots, X_{L}\right) \leq \prod_{\ell=1}^{L}\left(\frac{\beta_{\ell}}{\Omega_{\ell}}\right)^{\mu_{\ell}} \gamma_{0}^{L} \gamma_{\ell=1}^{L} \mu_{\ell} \alpha_{\ell} \\
& \times \exp \left(\sum_{\ell=1}^{L} \frac{\mu_{\ell}}{\beta_{\ell}}\left(\frac{x_{\ell}}{\gamma_{0}}\right)^{\alpha_{\ell}}\right) .
\end{aligned}
$$

Let $\eta_{0}=\max _{1 \leq \ell \leq L} \frac{\mu_{\ell}}{\beta_{\ell}}, \alpha_{\max }=\max _{1 \leq \ell \leq L} \alpha_{\ell}$, and $\alpha_{\min }=\min _{1 \leq \ell \leq L} \alpha_{\ell}$. We define the two sets

$$
I \triangleq\left\{\frac{x_{\ell}}{\gamma_{0}}: \frac{x_{\ell}}{\gamma_{0}}<1\right\} \text { and } J \triangleq\left\{\frac{x_{\ell}}{\gamma_{0}}: \frac{x_{\ell}}{\gamma_{0}} \geq 1\right\} .
$$

We can write

$$
\begin{aligned}
\sum_{\ell=1}^{L}\left(\frac{x_{\ell}}{\gamma_{0}}\right)^{\alpha_{\ell}} & =\sum_{\ell \in I}\left(\frac{x_{\ell}}{\gamma_{0}}\right)^{\alpha_{\ell}}+\sum_{\ell \in J}\left(\frac{x_{\ell}}{\gamma_{0}}\right)^{\alpha_{\ell}} \\
& \leq \sum_{\ell \in I}\left(\frac{x_{\ell}}{\gamma_{0}}\right)^{\alpha_{\min }}+\sum_{\ell \in J}\left(\frac{x_{\ell}}{\gamma_{0}}\right)^{\alpha_{\max }} .
\end{aligned}
$$


Using the embedding inequality of $L^{1}$ into $L^{\alpha}$ for $\alpha \geq 1$ [17], we can write, for any positive real numbers $\left\{v_{\ell}\right\}_{\ell=1}^{L}$

$$
\sum_{\ell=1}^{L} v_{\ell}^{\alpha} \leq\left(\sum_{\ell=1}^{L} v_{\ell}\right)^{\alpha}
$$

Since $\min _{1 \leq \ell \leq L} \alpha_{\ell}>1$, we use this inequality for $v_{\ell}=\frac{x_{\ell}}{\gamma_{0}}$, to get

$$
\begin{aligned}
\sum_{\ell=1}^{L}\left(\frac{x_{\ell}}{\gamma_{0}}\right)^{\alpha_{\ell}} & \leq\left(\sum_{\ell \in I} \frac{x_{\ell}}{\gamma_{0}}\right)^{\alpha_{\min }}+\left(\sum_{\ell \in J} \frac{x_{\ell}}{\gamma_{0}}\right)^{\alpha_{\max }} \\
& \leq\left(\sum_{\ell=1}^{L} \frac{x_{\ell}}{\gamma_{0}}\right)^{\alpha_{\min }}+\left(\sum_{\ell=1}^{L} \frac{x_{\ell}}{\gamma_{0}}\right)^{\alpha_{\max }} .
\end{aligned}
$$

Thus, we can write

$$
\begin{aligned}
& \mathcal{L}\left(X_{1}, \ldots, X_{L}\right) \leq \prod_{\ell=1}^{L}\left(\frac{\beta_{\ell}}{\Omega_{\ell}}\right)^{\mu_{\ell}} \gamma_{0}^{\sum_{\ell=1}^{L} \mu_{\ell} \alpha_{\ell}} \times \\
& \exp \left(\eta_{0}\left[\frac{1}{\gamma_{0}^{\alpha_{\min }}}\left(\sum_{\ell=1}^{L} x_{\ell}\right)^{\alpha_{\min }}+\frac{1}{\gamma_{0}^{\alpha_{\max }}}\left(\sum_{\ell=1}^{L} x_{\ell}\right)^{\alpha_{\max }}\right]\right) .
\end{aligned}
$$

Therefore, we obtain the following upper bound

$$
\begin{aligned}
& \mathbb{E}^{*}\left[\mathbb{1}_{\left\{S_{L} \leq \gamma_{0}\right\}} \mathcal{L}^{2}\left(X_{1}, \ldots, X_{L}\right)\right] \leq \prod_{\ell=1}^{L}\left(\frac{\beta_{\ell}}{\Omega_{\ell}}\right)^{2 \mu_{\ell}} \gamma_{0}^{2 \sum_{\ell=1}^{L} \mu_{\ell} \alpha_{\ell}} \\
& \times \exp \left(4 \eta_{0}\right)
\end{aligned}
$$

On the other hand, we have that

$$
\bigcap_{\ell=1}^{L}\left\{X_{\ell} \leq \frac{\gamma_{0}}{L}\right\} \subset\left\{\sum_{\ell=1}^{L} X_{\ell} \leq \gamma_{0}\right\} .
$$

In the i.n.i.d scenario, this leads to

$$
P \geq \prod_{\ell=1}^{L} \mathbb{P}\left(X_{\ell} \leq \frac{\gamma_{0}}{L}\right)=\prod_{\ell=1}^{L} F_{X_{\ell}}\left(\frac{\gamma_{0}}{L}\right) .
$$

We recall that the CDF of a GG RV is given by [2]

$$
F_{X_{\ell}}(x)=\frac{\gamma\left(\mu_{\ell}, \frac{\mu_{\ell}}{\Omega_{\ell}} x^{\alpha_{\ell}}\right)}{\Gamma\left(\mu_{\ell}\right)},
$$

where $\gamma(\cdot, \cdot)$ is the lower incomplete Gamma defined in [12, Eq. (8.350.1)]. Since we have $\gamma(s, z) \underset{z \rightarrow 0}{\sim} \frac{z^{s}}{s}$ [18, Eq. (2.272)], then we can write the following asymptotic expansion

$$
F_{X_{\ell}}\left(\frac{\gamma_{0}}{L}\right) \underset{\gamma_{0} \rightarrow 0}{\sim} \frac{\mu_{\ell}^{\mu_{\ell}-1} \gamma_{0}^{\alpha_{\ell} \mu_{\ell}}}{\Omega_{\ell}^{\mu_{\ell}} L^{\alpha_{\ell} \mu_{\ell}} \Gamma\left(\mu_{\ell}\right)}
$$

Thus, we get as $\gamma_{0} \rightarrow 0$

$$
\frac{1}{P^{2}} \leq \prod_{\ell=1}^{L}\left[\frac{\Omega_{\ell}^{\mu_{\ell}} L^{\alpha_{\ell} \mu_{\ell}} \Gamma\left(\mu_{\ell}\right)}{\mu_{\ell}^{\mu_{\ell}-1}}\right]^{2} \gamma_{0}^{-2} \sum_{\ell=1}^{L} \alpha_{\ell} \mu_{\ell}
$$

Combining (A.9) and (A.14), we obtain

$$
\begin{aligned}
& \limsup _{\gamma_{0} \rightarrow 0} \frac{\mathbb{E}^{*}\left[\mathbb{1}_{\left\{S_{L} \leq \gamma_{0}\right\}} \mathcal{L}^{2}\left(X_{1}, \ldots, X_{L}\right)\right]}{P^{2}} \\
& \leq \prod_{\ell=1}^{L}\left[\frac{\left[\Gamma\left(\mu_{\ell}\right)\right]^{1+\mu_{\ell} \alpha_{\ell}} \mu_{\ell}^{\alpha_{\ell} \mu_{\ell}-\mu_{\ell}+1}}{\left[\Gamma\left(\mu_{\ell}+\frac{1}{\alpha_{\ell}}\right)\right]^{\mu_{\ell} \alpha_{\ell}}}\right]^{2} \exp \left(4 \eta_{0}\right) .
\end{aligned}
$$

and hence the proof is concluded.

\section{REFERENCES}

[1] M. D. Yacoub, "The $\alpha-\mu$ distribution: A physical fading model for the stacy distribution," IEEE Transactions on Vehicular Technology, vol. 56, pp. 27-34, Jan 2007.

[2] E. W. Stacy, "A generalization of the gamma distribution," The Annals of Mathematical Statistics, vol. 33, pp. 1187-1192, 1962.

[3] M. K. Simon and M. S. Alouini, Digital Communications Over Fading Channels, Second Edition ed. New York: John Wiley and Sons, 2005.

[4] T. Piboongungon, V. A. Aalo, and C. D. Iskander, "Average error rate of linear diversity reception schemes over generalized Gamma fading channels," in Proceedings of IEEE Southeast Conference, 2005., Ft. Lauderdale, Fl, April 2005, pp. 265-270.

[5] A. Annamalai, C. Tellambura, and V. K. Bhargava, "Equal-gain diversity receiver performance in wireless channels," IEEE Transactions on Communications, vol. 48, pp. 1732-1745, Oct 2000.

[6] N. C. Sagias, P. T. Mathiopoulos, P. S. Bithas, and G. K. Karagiannidis, "On the distribution of the sum of generalized Gamma variates and applications to satellite digital communications," in Proceedings of 2nd International Symposium on Wireless Communication Systems, Siena, Italy, Sept 2005, pp. 785-789.

[7] D. B. D. Costa, M. D. Yacoub, and J. Filho, "Highly accurate closed-form approximations to the sum of $\alpha-\mu$ variates and applications," IEEE Transactions on Wireless Communications, vol. 7, pp. 3301-3306, September 2008.

[8] M. M. H. E. Ayadi and M. H. Ismail, "Novel closedform exact expressions and asymptotic analysis for the symbol error rate of single- and multiple-branch MRC and EGC receivers over $\alpha-\mu$ fading," IEEE Transactions on Vehicular Technology, vol. 63, pp. 4277-4291, Nov 2014.

[9] N. B. Rached, A. Kammoun, M.-S. Alouini, and R. Tempone, "Unified importance sampling schemes for efficient simulation of outage capacity over generalized fading channels," IEEE Journal of Selected Topics in Signal Processing, vol. 10, pp. 376-388, March 2016.

[10] C. B. Issaid, N. B. Rached, A. Kammoun, M.-S. Alouini, and R. Tempone, "On the sum of Gamma-Gamma variates with application to the fast outage probability evaluation over fading channels," Washington D.C., USA, Dec 2016, Accepted at the Global Conference on Signal and Information Processing (GlobalSIP'2016). 
[11] F. Yilmaz and M.-S. Alouini, "A unified MGF-based capacity analysis of diversity combiners over generalized fading channels," IEEE Transactions on Communications, vol. 60, pp. 862-875, Mar. 2012.

[12] I. S. Gradshteyn and I. M. Ryzhik, Table of Integrals, Series, and Products, Seventh Edition ed. Amsterdam: Elsevier/Academic Press, 2007.

[13] Y. Li, M. Piro, and V. Angelakisi, "Design of cellular backhaul topology using the FSO technology," in Proceedings of 2nd International Workshop on Optical Wireless Communications (IWOW), Newcastle, UK, Oct 2013, pp. 6-10.

[14] S. Hur, T. Kim, D. J. Love, J. V. Krogmeier, T. A. Thomas, and A. Ghosh, "Millimeter wave beamforming for wireless backhaul and access in small cell networks," IEEE Transactions on Communications, vol. 61, pp.
4391-4403, October 2013.

[15] S. Asmussen and P. W. Glynn, Stochastic Simulation : Algorithms and Analysis, ser. Stochastic modelling and applied probability. New York: Springer, 2007.

[16] C. B. Issaid, M.-S. Alouini, and R. Tempone, "Unified importance sampling approach for the outage probability evaluation of diversity receivers over generalized channels," Submitted to IEEE Transactions on Wireless Communications. [Online]. Available: http://repository. kaust.edu.sa/kaust/handle/10754/622646

[17] A. Villani, "Another note on the inclusion $l^{p}(\mu) \subset$ $l^{q}(\mu)$, , The American Mathematical Monthly, vol. 92, pp. 485-487, 1985.

[18] D. W. Bliss and S. Govindasamy, Adaptive Wireless Communications: MIMO Channels and Networks. Cambridge, U.K.: Cambridge Univ. Press, 2013. 